\title{
Tumeur odontogène adénomatoïde : une nouvelle observation
}

\section{Adenomatoid odontogenic tumour: a new case}

\author{
MUSTAPHA LAHLOU*, HANANE BENHALIMA*, NABILA TADLAOUI**, ABDENASSER LAZRAK*, MOHAMED KZADRI
}

\section{RÉSUMÉ}

Une volumineuse tumeur odontogène adénomatoïde, chez une patiente de 16 ans, est rapportée. Le siège maxillaire de la tuméfaction, l'évolution lente sur 3 ans et l'association avec une dent incluse, a fait évoquer initialement un kyste dentigère. C'est l'examen histologique de la pièce d'énucléation qui a redressé le diagnostic. L'accent est mis sur les caractéristiques épidémiologiques, cliniques, évolutives et thérapeutiques de la tumeur odontogène adénomatoïde et sur la nécessité d'un diagnostic précoce pour éviter des sacrifices osseux et dentaires comme en témoigne cette observation. (Med Buccale Chir Buccale 2005 ; 11:97-101).

mots clés: tumeur odontogène adénomatoïde, dent incluse, kyste dentigère.

\section{SUMMARY}

médecine buccale chirurgie buccale

VOL. $11, \mathrm{~N}^{\circ} 2$ 2005

page 97

One case of voluminous adenomatoid odontogenic tumour in 16 years old female patient is reported. Dentigerous cyst is the most probable primary diagnosis in fact of the upper left jaw painless swelling, the slow growing and the association with unerupted tooth. After surgical enucleation, histological examination revealed the lesion to be adenomatoid odontogenic tumour. The authors point out the features of this tumour, necessity of a early diagnosis and treatment to ovoid the needless sacrifice of teeth and bone for a completely benign tumour. (Med Buccale Chir Buccale 2005 ; 11 : 97-101).

key words: adenomatoid odontogenic tumour, impacted tooth, dentigerous cyst.

\footnotetext{
* * Service d'ORL et chirurgie maxillo-faciale, Hôpital des spécialités, CHU de Rabat-Maroc.

${ }^{* *}$ Laboratoire d'anatomie et cytologie pathologique, Hôpital des spécialités, CHU de Rabat- Maroc.
}

Demande de tirés à part:

Lahlou Mustapha, Service d'ORL et chirurgie maxillo-faciale, Hôpital des spécialités, BP 6220, Rabat Institut, Maroc. mmlahlou@hotmail.com 
La tumeur odontogène adénomatoïde (TOA) est une tumeur bénigne rare parmi les tumeurs maxillaires et elle est traitée par simple énucléation. $\mathrm{Ce}$ travail a pour but de décrire un nouveau cas de TOA volumineuse, en insistant sur l'intérêt d'un diagnostic précoce afin d'éviter des séquelles chirurgicales importantes pour une pathologie strictement bénigne.

\section{OBSERVATION}

II s'agit d'une patiente âgée de 16 ans, d'origine rurale, sans antécédents pathologiques particuliers, référée dans notre centre pour une tumeur maxillaire gauche évoluant depuis 3 ans. L'examen exobuccal montre une tuméfaction indolore intéressant la région génienne gauche (Fig. 1). A l'examen endobuccal, il existe une voussure dans le vestibule supérieur gauche, centrée

médecine

buccale

chirurgie

buccale

VOL. $11, \mathrm{~N}^{\circ} 2$ 2005

page 98 sur la région canine-prémolaire, en partie dépressible et recouverte d'une muqueuse saine. Cet examen a révélé, par ailleurs, l'absence de la canine supérieure gauche. La palpation combinée exo- et endobuccale confirme l'intégrité des parties molles péri-maxillaires. L'examen rhinoscopique antérieur révèle une obstruction nasale gauche par refoulement du cornet inférieur contre la cloison nasale. Les aires ganglionnaires cervicales sont libres et le reste de l'examen ORL et maxillo-facial ne révèle rien de particulier.

Une radiographie en incidence de Blondeau montre une opacité de la totalité du sinus maxillaire gauche avec une image intrasinusienne rappelant la canine incluse (Fig. 2) ; l'examen tomodensitométrique une lésion hypodense, uniloculaire, bien circonscrite, mesurant $7 \times 5 \mathrm{~cm}$, comportant une dent incluse, bombant dans le sinus maxillaire et refoulant la cloison intersinusonasale vers la ligne médiane (Fig. 3). Cet aspect radio-clinique fait avant tout évoquer un kyste dentigère. Le traitement a consisté en une exérèse de cette néoformation, sous anesthésie générale et par une voie d'abord vestibulaire. Après résection de la corticale externe soufflée, une énucléation complète du kyste est réalisée avec avulsion des dents situées au pourtour de la tumeur (la 24 et 25).

La pièce d'exérèse comporte une partie charnue et blanchâtre, mesurant $2 \times 2 \mathrm{~cm}$, adhérente à une dent, et une partie kystique mesurant $4,5 \times 3 \mathrm{~cm}$. A l'examen microscopique, la formation kystique est bordée de cellules cubiques régulières. La prolifération tissulaire solide est faite de cellules épithéliales arrondies ou cuboïdes, de petite taille, avec un cytoplasme peu abondant et un noyau régulier ovalaire ou arrondi. Des cellules sont organisées en amas ou en nappes, et elles forment par endroits des tourbillons ou des structures glandulaires. On note, par ailleurs, une population minoritaire de cellules de petite taille avec

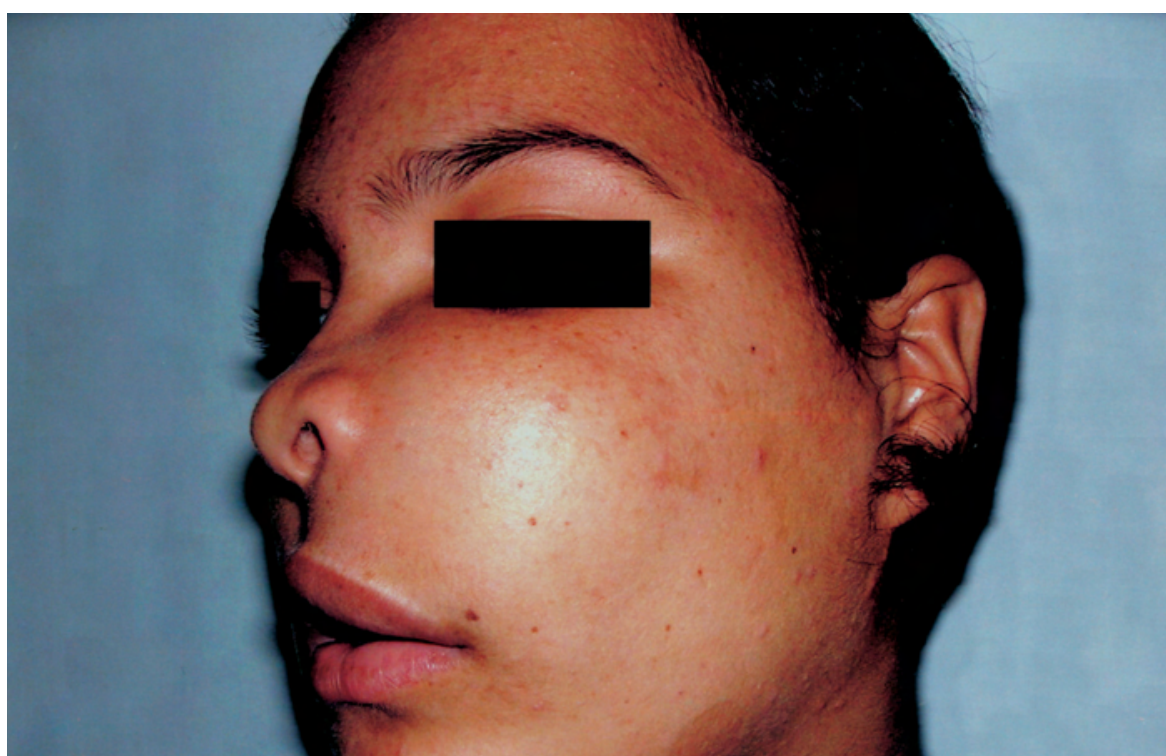

Figure 1 : Vue de face : tuméfaction génienne gauche. Photography of the face: left jaw swelling. 
Figure 2 : Radiographie en incidence Blondeau : opacité se projettant sur le sinus maxillaire gauche où siège une dent incluse.

Blondeau radiography: radiopacities of left maxillary sinus with impacted tooth.
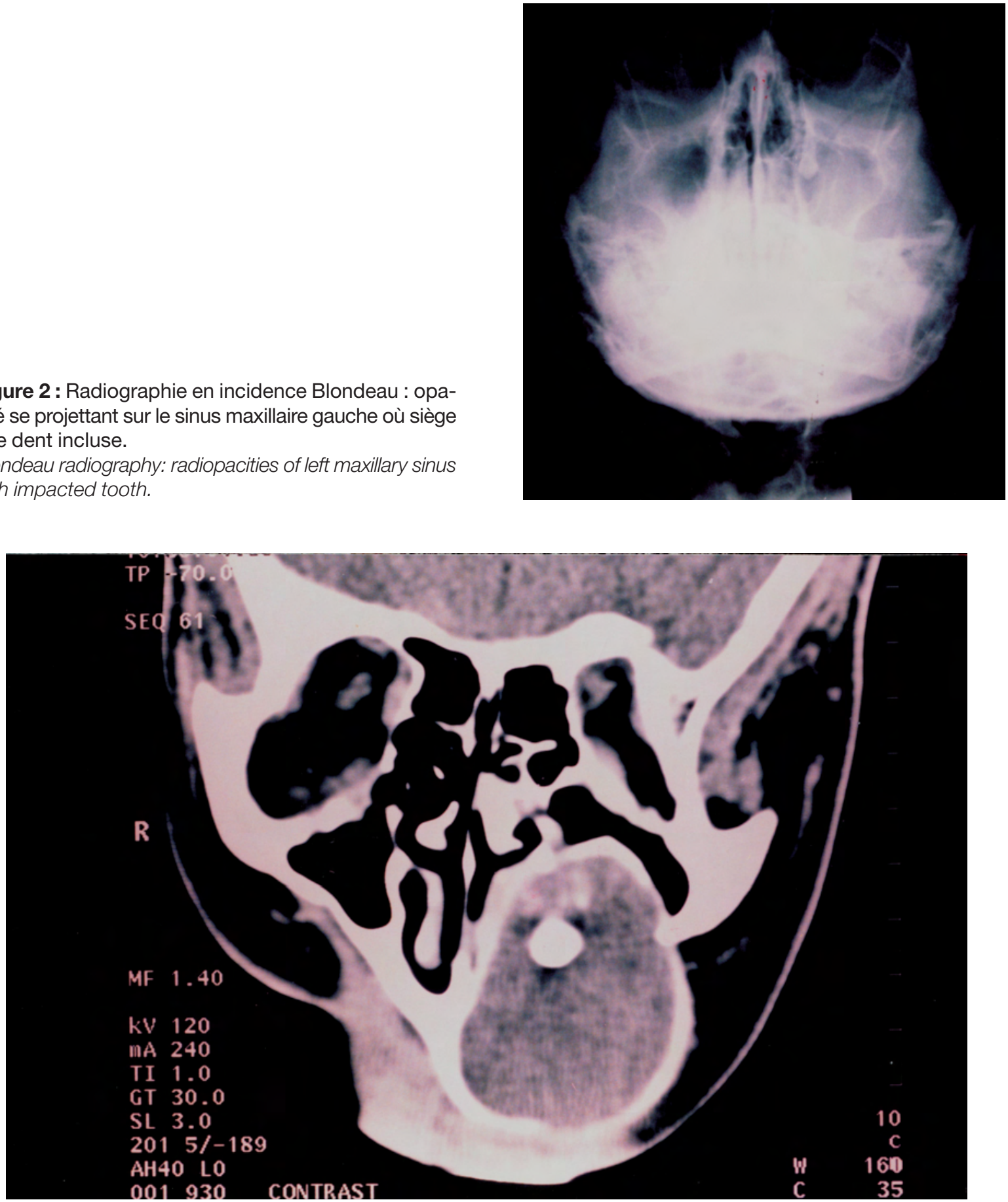

médecine

buccale

chirurgie

buccale

VOL. $11, \mathrm{~N}^{\circ} 2$

2005

page 99

Figure 3 : TDM en coupe coronale et en fenêtre parenchymateuse : formation hypodense, entourée d'une paroi hyperdense, contenant une dent.

CT coronal view: hypodense mass with hyperdense wall, containing a tooth.

des noyaux hyperchromatiques. Le stroma est moyennement vascularisé. L'ensemble correspond à la description classique d'une TOA (Fig. 4). Les suites postopératoires sont simples. Au der- nier contrôle, 12 mois après l'intrevention, il n'y a pas de signes de récidive et la patiente n'envisage pas le remplacement prothétique des dents extraites. 
médecine buccale chirurgie buccale

VOL. $11, \mathrm{~N}^{\circ} 2$ 2005

page 100

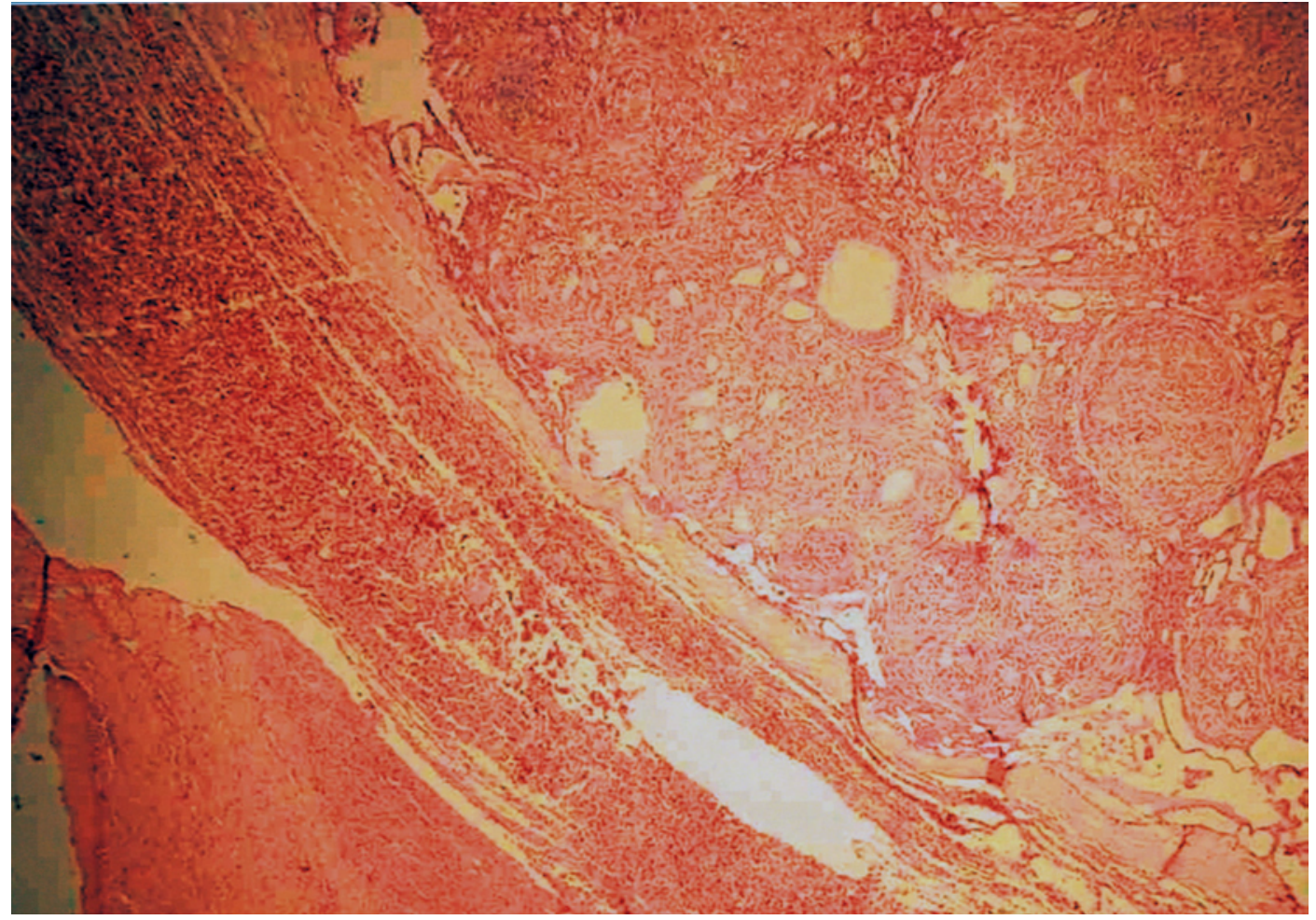

Figure 4 : Aspect histologique de la TOA : la tumeur est bien circonscrite, la proliferation épithéliale est constituée de petites cellules formant des travées, des tourbillons et des aires glandulaires (hémateine- éosine, $x$ 40). Histologic feature of adenomatoid odontogenie tumour: The tumor is wall circunscribed. The epithelial proliferation consists of small cells present in sheets, whorled and glandular areas (hematein-eosin, $x 40$ ).

\section{COMMENTAIRES}

Dans la dernière classification de l'Organisation Mondiale de Santé, la TOA est une tumeur intègrée dans le groupe des tumeurs odontogéniques épithéliales et conjonctives avec ou sans formation du tissu dentaire dur [1]. Elle est aussi connue improprement sous le terme d'adénoaméloblastome ou de tumeur adénoïde améloblastique [2]. Cette tumeur représente seulement $0,1 \%$ des kystes et tumeurs des maxillaires et $3 \%$ des tumeurs odontogènes [3, 4].

L'âge et le sexe, dans le cas rapporté, correspondent parfaitement aux données de la littérature. La TOA se rencontre surtout au cours de la deuxième décade (âge moyen de 19 ans) et les femmes sont plus touchées que les hommes avec un sexe ratio de $2 / 1$ ou $3 / 1$ [4,5,6]. La partie antérieure du maxillaire, et particulièrement la région canine, représente le siège de prédilection de la tumeur [5, 7], l'atteinte mandibulaire est moins fré- quente et représente le tiers des cas environ [2]. L'association avec une dent incluse, notamment la canine, est souvent rapportée $[4,6,7]$.

La TOA présente souvent une évolution prolongée, lente et indolente. Elle est souvent découverte lors d'un examen dentaire de routine ou d'investigations pour un retard d'éruption dentaire [4]. La taille de la TOA varie de 1,5 à $3 \mathrm{~cm}$, mais elle peut atteindre, parfois, un volume plus important [5] et être responsable de déplacements dentaires, de troubles fonctionnels et/ou esthétiques [6] comme ceux observés dans le cas présenté où la négligence et les difficultés d'accès aux soins expliquent, en partie, le diagnostic tardif.

A la radiographie, la TOA donne une image radiotransparente, uniloculaire, bien circonscrite [2,8]. La présence parfois de fines calcifications peut orienter le diagnostic [4,5,6]. Lorsqu'une dent incluse est associée, elle sera comprise en totalité (couronne et racine) dans la cavité kystique [9]. 
Les dents voisines peuvent être déplacées si la TOA est volumineuse mais les racines ne sont normalement pas résorbées [6]. Devant cette importante tuméfaction faciale accompagnée d'une obstruction de la fosse nasale homolatérale, un examen tomodensitométrique a été réalisé pour apprécier l'extension de la TOA et guider le geste opératoire.

L'aspect histologique, observé dans ce cas, est très caractéristique de la TOA. Le diagnostic différentiel intéresse essentiellement l'aspect radiologique et fait évoquer avant tout un kyste dentigère; le kyste s'insère au collet de la dent

\section{RÉFÉRENCES}

1 - Kramer IRH, PIndBorg JJ, Shear M. International histological classification of tumours: histological typing of odontogenic tumours (2nd ed) (pp 7-9). Springer-Verlag, Berlin, Heidelberg, 1992.

2 - PANtoja R, Delaire J. Une nouvelle observation de tumeur odontogénique adénomatoïde. Rev Stomatol Chir Maxillofac 1991; 92 : 98-100.

3 - RegezI JA, KerR DA, Courtney RM. Odontogenic tumours: Analysis of 706 cases. J Oral. Surg 1978 ; 36 : 771-8.

4 - Precious DS, Delaire J, Wright P, Landry P. La tumeur odontogénique adénomatoïde. Rev. Stomatol Chir Maxillofac 1984 ; 85 : 472-6.

5 - Dayı E, Gürbüz G, Bilge OM, Ciftcioglu MA. Adenomatoid odontogenic tumour (adenoameloblastoma). Case report and review of the literature. Aust Dent $1997 ; 42: 315-8$. incluse et seule la couronne est intrakystique, au moins au stade initial [6,9]. D'autres tumeurs peuvent prêter à confusion avec la TOA : l'améloblastome, le carcinome adénoïde kystique et le carcinome mucoépidérmoïde [6].

Le traitement de la TOA est simple et consiste en une énucléation complète. Aucun cas de récidive ou de transformation maligne n'a été rapporté $[2,4,5]$.

La surveillance du développement dentaire permet de faire un diagnostic précoce, évitant ainsi d'éventuels sacrifices osseux et dentaires pour une pathologie aussi bénigne.
6 - Pecheur A, De Clercq D, Reychler H. La tumeur odontogénique adénomatoïde. Rev Stomatol Chir Maxillofac $1992 ; 93: 341-4$.

7 - Tolda M, Hyodo I, OkUda T, Tatematsu N. Adenomatoid odontogenic tumour: Report of two cases and survey of 126 cases in Japan. J Oral Maxillofac. Surg 1990 ; 48 : 404-8.

8 - GIANSANTI JS, SOMEREN A, WaldRon CA. Adenomatoid odontogenic tumour (adenoameloblastoma). Survey of 3 cases. Oral Surg Oral Med Oral Pathol 1970 ; 30 : 6988.

9 - Geist SY, MaLlon HL. Adenomatoid odontogenic tumour. Report of an unusually large lesion in the mandibule. J Oral Maxillofac Surg 1995 ; 53 : 714-7. médecine buccale chirurgie buccale

VOL. $11, \mathrm{~N}^{\circ} 2$ 2005

page 101 